\title{
Arrêt des anticoagulants avant un acte de chirurgie buccale : mythes et réalités
}

\author{
Anticoagulant withdrawal before oral surgery: mythis and realities
}

\author{
SOUKĖYE DIA TINE ${ }^{1}, 3$, OUMY SECK ${ }^{1}$, NDĖYE FATOU KÉBÉ1 , NDĖYE FATOU DIENG ${ }^{1}$, \\ IBRAHIMA CISSÉ 1 , ABDOUL KANE², BOUBACAR DIALLO ${ }^{3}$
}

\section{RÉSUMÉ}

Il s'agit d'une étude transversale et descriptive portant sur une période de 9 mois (janvier à septembre 2005). Elle a eu pour cadre le service d'Odontologie d'un centre hospitalier public de la région de Dakar (Sénégal). Elle a concerné 32 patients référés au service d'Odontologie par le Service de Cardiologie. Ils présentaient une pathologie cardio-vasculaire ayant nécessité la prescription d'un anti-vitamine $\mathrm{K}$ et ils devaient subir un acte de chirurgie buccale.

L'objectif du travail était de déterminer la valeur de l'INR (International Normalized Ratio) permettant de réaliser un acte de chirurgie buccale sans risque hémorragique.

Nous avons exploité les paramètres suivants : identification du malade, état bucco-dentaire, type de soins bucco-dentaires, type de cardiopathies, posologie des AVK, valeur de l'INR, moyens d'hémostase.

$81,25 \%$ (26 sur 32 ) des patients étaient des femmes, soit un sexe ratio $M / F$ de 0,23 . La moyenne d'âge était de 53 ans, avec des extrêmes de 21 ans et 84 ans. 56,25\% des patients ont subi une seule extraction, $25 \%$ des extractions multiples et $18,75 \%$ ont bénéficié d'un détartrage-curetage.

La valeur moyenne de l'INR est de 2,38 avec une variance de 0,07 et un écart type de 0,27.

Grâce aux moyens d'hémostase instaurés, aucune complication hémorragique postopératoire n'est survenue. Il semble bien que le risque d'accident thromboembolique à l'arrêt du traitement est beaucoup plus préjudiciable que le risque hémorragique pour le patient. Med Buccale Chir Buccale 2008 ; 14 : 103-109.

mots clés : anticoagulants, risque hémorragique, chirurgie buccale, cardiopathies, hémostase.

\section{SUMMARY}

The study is transversal and descriptive. It take place in the odontology department in a public hospital in Dakar (Senegal), during 9 month (January - September 2005).

It concerned 32 patients referred to the Odontology service by the Cardiology service. They presented a cardiovascular pathology, having required an anticoagulant therapy, and also need an act of oral surgery. The objective of this work was to determine the INR value (International Normalized Ratio) making possible to carry out an act of oral surgery without haemorrhagic risk. We exploited the following parameters: identification of the patient, oral state, type of oral surgery, type of cardiopathy, AVK posology, INR value, means haemostasis. 
$81,25 \%$ (26 on 32) of the patients were women, with a sex ratio M/F of 0,23. The average age was 53 years, with 21 and 84 years like the extremes. 56,25\% of our patients had undergone a single extraction, 25\% had beneficed multiple extractions, and 18,75\% had profited from a scaling-curetting.

The average value of the INR was 2,38 with an variance of 0,07 and an "ecart type" of 0,27

In favour of the haemostat means, no haemorrhagic complication post operational was revealed. It is allowed that the risk of thromboembolic accident by stopping the treatment is much more prejudicial than the hemorrhagic risk for the patient. Med Buccale Chir Buccale 2008; 14: 103-109.

key words: anticoagulants, hemorrhagic risk, oral surgery, cardiopathy, haemostasis.

médecine buccale chirurgie buccale

VOL. $14, \mathrm{~N}^{\circ} 2$ 2008

page 104
Avant d'effectuer un acte de chirurgie buccale, le praticien est souvent préoccupé par le risque hémorragique chez les patients sous anticoagulants. II se pose souvent la question de la poursuite ou non du traitement : l'arrêt transitoire est susceptible de limiter le risque hémorragique mais il augmente le risque thromboembolique.

Les données actuelles de la littérature montrent que le risque d'accident thromboembolique lié à l'arrêt ou à la modification du traitement anticoagulant, est beaucoup plus préjudiciable que le risque hémorragique lié à l'acte [1]. L'arrêt du traitement anticoagulant pour un acte de chirurgie buccale est très controversé actuellement.

Cette étude avait pour objectif de déterminer la valeur de l'INR (International Normalized Ratio) permettant de réaliser un acte de chirurgie buccale sans risque hémorragique dans notre pratique.

\section{MATERIEL ET METHODE}

II s'agit d'une étude transversale et descriptive, qui s'est déroulée sur une période de neuf mois (janvier à septembre 2005). Elle a eu pour cadre les services de Cardiologie et d'Odonto-stomatologie de I'Hôpital Général de Grand Yoff (HOGGY) de Dakar. Elle a concerné 32 patients traités dans le service de Cardiologie, sous anticoagulants, devant subir un acte de chirurgie buccale dans le service d'Odonto-stomatologie. Des fiches individuelles de collecte des données ont été établies afin d'exploiter les paramètres suivants : identification du malade, état bucco-dentaire, type de cardiopathies, posologie des AVK, valeur de l'INR, type de chirurgie buccale, moyens d'hémostase.
Avant tout acte chirurgical, le patient devait avoir un INR compris entre 2 et 3 et le dernier examen de I'INR devait se faire moins de 24 heures avant l'intervention. L'hémostase était assurée par une compression immédiate selon la méthode de Descrozailles [2]. Elle était de 3 types :

- Compression intrinsèque ou tamponnement alvéolaire : elle a consisté à introduire, dans l'alvéole, un matériau hémostatique résorbable, comme une éponge de gélatine (Hémofibrine $®)$ ) ou une mèche d'oxycellulose (Surgicel $\circledast$ ), en tassant de la profondeur à la surface. Puis on demande au patient de mordre fortement sur une compresse de gaz stérile pendant 10 à 15 minutes.

- Compression extrinsèque : on réalise d'abord une compression manuelle forte sur la gencive ou sur l'alvéole avec une compresse de gaz stérile. Puis on demande au patient de mordre fortement sur la compresse ou de serrer fortement les lèvres pendant 10 à 15 minutes.

- Tamponnement mixte : après tamponnement de l'alvéole avec de l'eau oxygénée à 10 \% (peroxyde d'hydrogène), une mèche d'oxycellulose (Surgicel囚) est introduite et tassée dans l'alvéole. Des points de suture permettront de maintenir le matériau et d'assurer l'hémostase qui sera complétée par une compression extrinsèque.

Après chaque extraction, le patient est gardé en observation pendant 15 minutes dans la salle d'attente en lui demandant de mordre sur une compresse. Après ce délai, on vérifie et on évalue le saignement. Une semaine après l'extraction, le patient est revu pour apprécier la cicatrisation de la plaie. Lors de l'intervention, nous disposons des moyens nécessaires pour réaliser une hémostase efficace (eau oxygénée, éponge de gélatine, mèche d'oxycellulose, fils de suture...). 


\section{RESULTATS}

Répartition des malades selon le sexe (Fig.1) $81,25 \%(26)$ des patients étaient des femmes contre $18,75 \%$ (6) d'hommes, soit un sexe ratio $\mathrm{M} / \mathrm{F}$ de 0,23 .

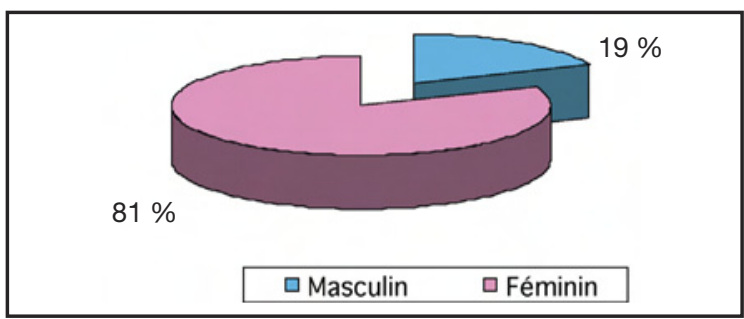

Figure 1 : Répartition des 32 malades selon le sexe. Distribution of 32 cases by sex.

\section{Répartition des malades selon l'âge}

La tranche d'âge la plus représentée se situait entre 21 et 30 ans, soit $21,87 \%$ des patients. La moyenne d'âge était de 53 ans avec des extrêmes de 21 et 84 ans (Fig. 2).

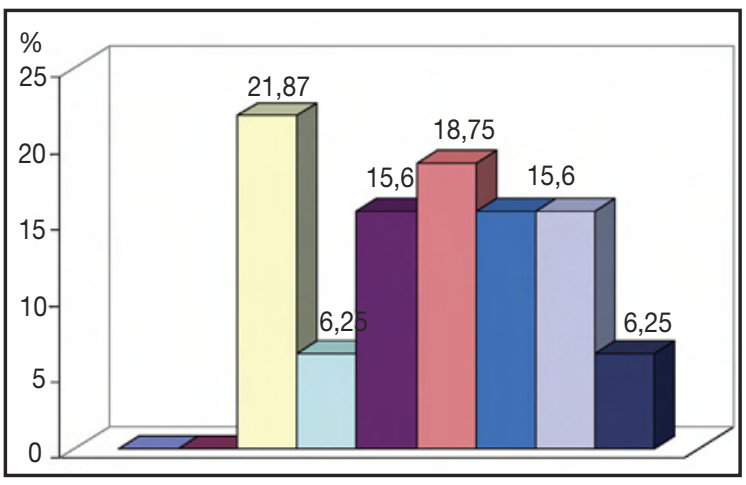

Figure 2 : Répartition des 32 malades selon la tranche l'âge.

Distribution of 32 cases by age.

Répartition des malades selon l'état buccodentaire (Fig. 3)

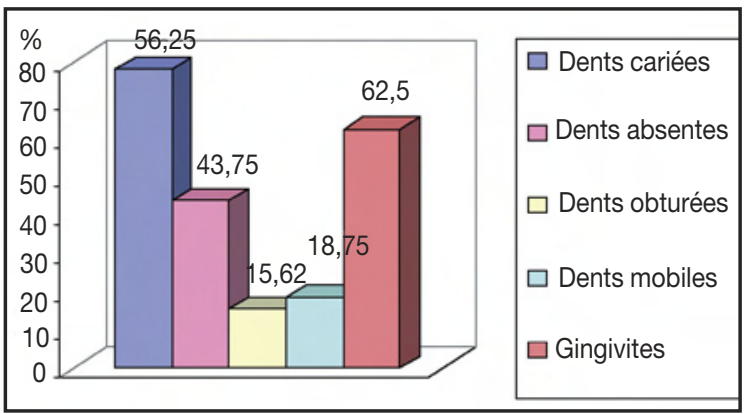

Figure 3 : Répartition des 32 malades selon l'état bucco-dentaire.

Distribution of 32 cases by oro-dental status.
Répartition des malades selon le type de chirurgie buccale (Fig. 4)

Plus de la moitié des patients ont subi une seule extraction (56,25\%) ; ceux qui ont subi des extractions multiples dans la même séance représentaient $25 \%$, et $18,75 \%$ ont bénéficié d'un détartrage-curetage à l'aveugle.

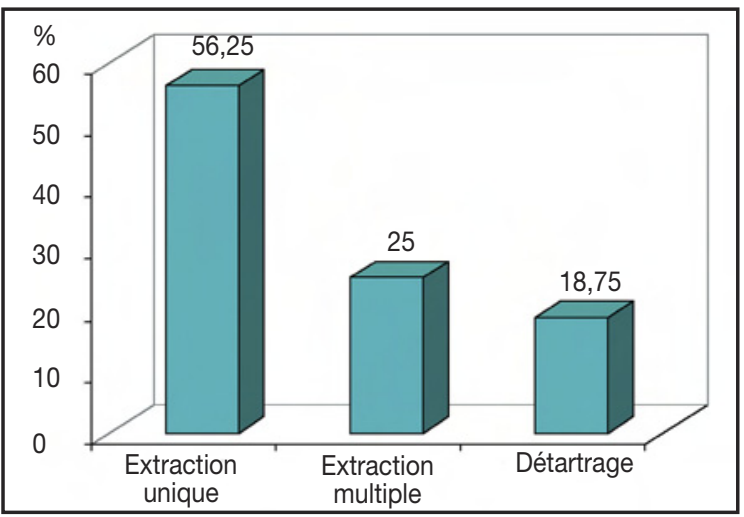

Figure 4 : Répartition des 32 malades selon le type de traitement chirurgico-dentaire.

Distribution of 32 cases by dental treatment.

\section{Répartition des malades selon la posologie de I'AVK}

L'acénocoumarol (Sintrom $®)$ était l'AVK utilisé chez tous les patients, par voie orale, à une posologie journalière adaptée pour chaque patient. II était préscrit par le service de Cardiologie de I'HOGGY : $62,5 \%$ des patients avaient une posologie de $2 \mathrm{mg}$ par jour, 12,5\% $1 \mathrm{mg}$ par jour, 21,87 \% 3 mg par jour, 3,12 \% 4 mg par jour.

Répartition des malades selon le type de pathologie cardiovasculaire (Fig. 5)

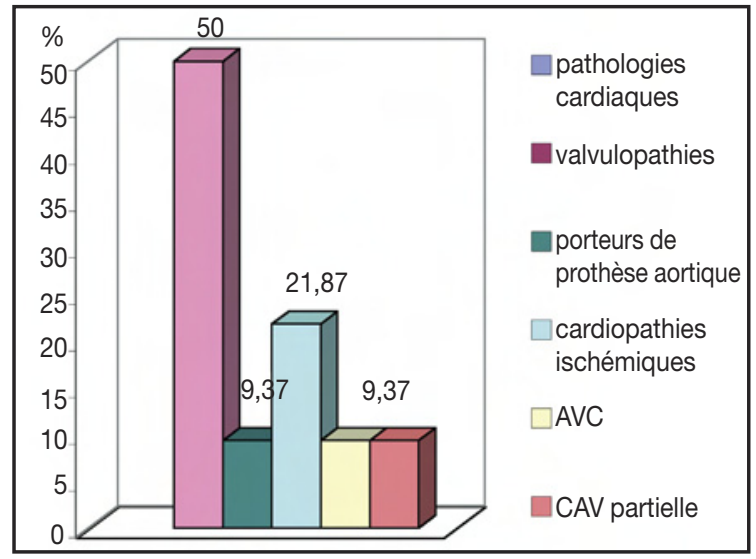

Figure 5 : Répartition des 32 malades selon le type de pathologie cardiaque.

Distribution of 32 cases by cardiac pathologies. médecine

buccale

chirurgie

buccale

VOL. $14, \mathrm{~N}^{\circ} 2$ 2008

page 105 
médecine

buccale

chirurgie

buccale

VOL. $14, \mathrm{~N}^{\circ} 2$ 2008

page 106
$50 \%$ des patients présentaient une valvulopathie, $21,87 \%$ une cardiopathie ischémique. $9,37 \%$ étaient porteurs d'une prothèse aortique et 9,37\% avaient été victimes d'accidents vasculo-cérébraux (AVC) de même que ceux présentant un canal artrio-ventriculaire (CAV) partiel.

\section{Répartition des patients selon l'INR, le type de chirurgie et le type d'hémostase (Tab. 1)}

La valeur moyenne de l'INR est de 2,38 avec une variance de 0,07 et un écart type de 0,27.

Tableau 1 : Répartition des 32 patients selon l'INR, le type de traitement odonto-stomatologique et les moyens d'hémostase.

Distribution of 32 cases by INR, dental treatment and technic hemostasis.

\begin{tabular}{|c|c|c|}
\hline INR & Type de traitement odontologique & Moyens d'hémostase \\
\hline 2 & Extraction unique & Compression extrinsèque \\
\hline 2 & Détartrage & Compression extrinsèque \\
\hline 2,04 & Extraction unique & Compression extrinsèque \\
\hline 2,04 & Extraction unique (alvéolectomie) & Tamponnement mixte \\
\hline 2,1 & Extraction unique & Compression extrinsèque \\
\hline 2,12 & Détartrage + curetage & Compression extrinsèque \\
\hline 2,13 & Extraction unique & Compression extrinsèque \\
\hline 2,15 & Extraction unique & Compression extrinsèque \\
\hline 2,19 & Détartrage & Compression extrinsèque \\
\hline 2,19 & Extraction multiple + détartrage & Tamponnement mixte \\
\hline 2,2 & Détartrage & Compression extrinsèque \\
\hline 2,21 & Extraction multiple & Tamponnement mixte \\
\hline 2,21 & Extraction unique & Compression extrinsèque \\
\hline 2,29 & Extraction multiple & Tamponnement mixte \\
\hline 2,30 & Extraction multiple & Tamponnement mixte \\
\hline 2,31 & Extraction unique & Compression extrinsèque \\
\hline 2,34 & Détartrage & Compression extrinsèque \\
\hline 2,31 & Extraction unique & Compression extrinsèque \\
\hline 2,38 & Extraction multiple & Tamponnement mixte \\
\hline 2,39 & Extraction unique & Compression intrinsèque \\
\hline 2,4 & Extraction multiple & Tamponnement mixte \\
\hline 2,59 & Extraction multiple & Tamponnement mixte \\
\hline 2,69 & Extraction unique & Compression intrinsèque \\
\hline 2,69 & Extraction unique & Compression intrinsèque \\
\hline 2,7 & Extraction unique & Compression intrinsèque \\
\hline 2,71 & Extraction multiple & Tamponnement mixte \\
\hline 2,71 & Extraction multiple & Tamponnement mixte \\
\hline 2,74 & Extraction unique & Compression intrinsèque \\
\hline 2,75 & Extraction multiple & Tamponnement mixte \\
\hline 2,75 & Extraction unique & Compression intrinsèque \\
\hline 2,83 & Extraction multiple & Tamponnement mixte \\
\hline 2,84 & Extraction multiple & Tamponnement mixte \\
\hline
\end{tabular}

L'hémostase était assurée par une compression immédiate qui était de type :

- Intrinsèque ou tamponnement alvéolaire dans 6 cas d'extraction unitaire dont l'INR était $>2,39$.

- Extrinsèque chez 13 patients ayant bénéficié d'un détartrage ou d'une extraction unitaire avec un INR $<2,39$.

- Tamponnement mixte dans les 13 cas d'extractions multiples quelque soit la valeur de l'INR. 106 


\section{DISCUSSION}

Nous avons rencontré quelques difficultés dans la sélection des patients car il fallait avoir un INR stable compris entre 2 et 3 , et effectué moins de 24 heures avant l'acte chirurgical. Ce taux d'INR étant considéré comme la valeur thérapeutique efficace pour prévenir l'accident thromboembolique dans le service d'Odonto-stomatologie.

Le faible niveau socio-économique et le manque de motivation pour les soins dentaires des patients expliquent en grande partie le recrutement de seulement 32 cas répondant aux critères de sélection en 9 mois. La majorité des patients sont des femmes, 26 cas soit $81 \%$. Au Sénégal, la prédominance des femmes, chez les patients atteints de maladies cardio-vasculaires a été décrite dans plusieurs études, par exemple celle de Diallo ${ }^{[3]}$ qui a constaté un pourcentage que $70 \%$ des cas concernaient des patientes. La tranche d'âge la plus représentée se situe entre 21 et 30 ans, soit 21, $87 \%$ de l'échantillon. L'âge des malades varie entre 21 ans et 84 ans. Au Sénégal, les valvulopathies rhumatismales sont nettement prédominantes, contrairement à ce que l'on observe dans les pays développés où les formes dégénératives liées à l'âge sont plus fréquentes. Cet aspect serait lié, dans cette tranche d'âge et dans des couches sociales défavorisées, à la fréquence de l'angine qui, lorsqu'elle est mal traitée, peut être responsable du rhumatisme articulaire aigu (RAA) et des valvulopathies rhumatismales ${ }^{[3]}$. Les caries sont très fréquentes (78\%). Cette prévalence chez les sujets jeunes met en évidence l'aspect juvénile de la carie dentaire et de ses complications. En effet la mauvaise hygiène et l'insuffisance de brossage chez les jeunes patients, associée à une forte tendance à la prise d'aliments riches en hydrates de carbone, provoquent l'apparition de lésions carieuses pouvant évoluer vers des pathologies locorégionales graves comme l'infection focale [4].

L'extraction dentaire a constitué l'acte chirurgical prédominant, $81 \%$ des patients ont subi au moins une extraction dentaire. Cet aspect pourrait être expliqué par le retard de prise en charge car les patients ont tendance à négliger leur pathologie bucco-dentaire.
Lors de l'intervention, la prévention des complications hémorragiques a été de rigueur. L'extraction doit être la moins traumatisante possible pour éviter une hémorragie per- ou postopératoire ; il convient d'agir avec précaution afin d'éviter toute dilacération des tissus ou une fracture alvéolaire ${ }^{[5,6]}$.

L'acénocoumarol (Sintrom $®)$ ) a été la seule molécule utilisée comme anticoagulant chez tous les patients de notre série. L'efficacité du traitement anticoagulant par les anti-vitamines K est appréciée en pratique courante par la valeur thérapeutique de l'INR estimée entre 2 et 3 [7,8]. Les valeurs faibles de la variance 0,07 et de l'écart type 0,27 montrent que la dispersion du taux de l'INR est étroite et peu significative autour de la moyenne qui est de 2,38. Ces valeurs thérapeutiques d'INR adoptées par le service de Cardiologie ont permis de réaliser les différents actes de chirurgie buccale de notre série sans faire modifier ou arrêter le traitement anti-coagulant.

De nombreuses études ont mise en évidence la possibilité de pratiquer les interventions de chirurgie bucco-dentaire sans interrompre ou diminuer la posologie des anti-coagulants à condition que l'INR soit stable et inférieur à 4 et que le protocole opératoire soit rigoureux avec maîtrise des moyens d'hémostase locale [9]. $50 \%$ des cas sont constitués par des valvulopathies dont les accidents thrombo-emboliques représentent une des complications les plus redoutables d'où la nécessité d'un traitement anticoagulant continu, efficace et bien surveillé. La prise en charge des cardiopathies ischémiques nécessite aussi un traitement anti-thrombotique (antiagrégant plaquettaire, anticoagulant).

Dans $75 \%$ des cas, on a eu recours à la compression extrinsèque ou intrinsèque uniquement contre $25 \%$ au tamponnement mixte pour les extractions multiples ayant nécessité des sutures. Pour toutes les extractions et détartrages effectués, aucune complication hémorragique majeure n'a été relevée. Pourtant aucune prescription d'hémostatique à usage général n'a été effectuée au préalable. Les moyens d'hémostase locale de par leur simplicité et leur efficacité doivent reléguer au second plan les traitements hémostatiques par voie générale [10,11]. médecine

buccale

chirurgie

buccale

VOL. $14, \mathrm{~N}^{\circ} 2$ 2008

page 107 
médecine buccale chirurgie buccale

VOL. $14, \mathrm{~N}^{\circ} 2$ 2008
Compte tenu des graves risques associés à l'arrêt de l'anticoagulant, il faudrait revoir la pratique qui vise généralement à interrompre l'administration des anti-coagulants avant un acte de chirurgie buccale [5, 12].

D'après un essai randomisé réalisé aux USA par Troulis ${ }^{[13]}$, ayant porté sur 109 patients traités sous AVK et dont l'INR se situait dans la marge thérapeutique normale (entre 2 et 3), l'extraction dentaire pouvait être pratiquée sans modifier l'anticoagulothérapie orale. Dans le cadre d'une étude plus exhaustive, effectuée par Wahl [14], 249 patients ayant subi au total 543 extractions dentaires ont été répartis en 5 groupes, selon la valeur de l'INR le jour de l'intervention. Aucune différence significative n'a été observée entre eux. Dans notre série, l'hémostase locale a suffit pour prévenir le saignement post-opératoire dans tous les cas. Cependant en milieu hospitalier il est nécessaire de disposer de colles biologiques pour juguler efficacement toute hémmorragie buccale qui ne serait pas contrôlée par des moyens simples.

\section{RÉFÉRENCES}

1 - Levesque H, Peron JM. Antiagrégants plaquettaires et antivitamines $\mathrm{K}$ en stomatologie et chirurgie maxillo-faciale. Rev Stomatol Chir Maxillofac 2003; $104: 80-90$

2 - Descrozailles $\mathrm{CH}$, Drouillat JP. Le risque hémorragique chez les cardiaques. Actual Odonto-stomatol $1978 ; 32$ : 245-82.

3 - Diallo M. Attitudes du chirurgien dentiste devant un malade atteint de troubles cardio-vasculaires. Thèse de chirurgie dentaire. Dakar, 1997, n²3.

4 - Kolokotronis A. Connaissances actuelles sur la flore buccale et son éventuelle implication dans l'étiopathogenie de l'infection a distance (p. 167). Quintessence du Congrès de l'Association dentaire française 2005.

5 - Samson J, Bernard JP, Scheffer P. Avulsions dentaires et troubles de l'hémostase. Inf Dent 1982 ; 64 : 161-5.

6 - Evans BE, Irving SP, Aledort LM. Can anticoagulant be continued diuring dental extraction. Results of a randomized controlled trial. Br J Oral Maxillofac Surg 2002 ; $40: 248-52$.
II ressort de ces analyses que les cas documentés de complications emboliques sont plus importants que les cas documentés de complications hémorragiques $[5,15]$. II est admis que le risque hémorragique après une chirurgie buccale est minime par rapport au risque thromboembolique [16].

\section{CONCLUSION}

L'évaluation clinique des risques de saignement après un acte de chirurgie buccale chez des patients sous anticoagulants montre qu'il n'est pas nécessaire d'interrompre ou de modifier le traitement.

Cependant il faut maîtriser les mesures d'hémostase locale appropriées et avoir une bonne appréciation de l'état de la coagulabilité du patient par rapport à l'importance de l'acte chirurgical. Toutefois, si l'INR est supérieur à 3, le patient doit être traité en milieu hospitalier et la collaboration avec le médecin traitant est capitale.
7 - Troulis MJ, Head TW, Leclerc JP. What is the INR? J Can Dent Assoc 1996 ; 62 : 428-30.

8 - Souto JC, Olivier Z, Azuazu ZI, Vwes A, Foutauberto J. Oral surgery in anticoagulated patients without reducing the dose of oral anticoagulant : a prospective randomized study. J Oral Maxillofac Surg $1996 ; 54: 27-32$

9 - Société francophone de médecine buccale et chirurgie buccale et Société française de cardiologie. Recommandations pour prise en charge des patients sous anti-vitamines $\mathrm{K}$ en chirurgie bucco-dentaire. Med Buc Chir Buc 2006 : 4.189-211.

10 - Blinder DD, Manor Y, Martinowitz U, Taicher S, Hasliomer T. Dental extraction in patients maintained on continued oral anticoagulant: comparison of local haemostatic modalities. Oral Surg Oral Med Oral Pathol Oral Radiol Endod 1999, 88 : 137-40.

11 - Cautaloube D, Rives JM, Daize E, Jalleras JP. Traitement préventif et curatif des hémorragies opératoires. EMC Stomatologie Odontologie 22030-C, Paris, 1991. 
12 - Société française de cardiologie. Recomman-dations de la société française de cardiologie concernant les indications et la surveillance du traitement anticoagulant oral. Arch Mal Cœur Vaiss 1997 ; 90 : 1289305.

13 - Troulis MJ, Head TW, Leclerc JP. Dental extraction in patient on oral anticoagulant: a survey of practices in North America. J Oral Maxillofac Surg 2000; 58 : 131-5.

14 - Wahl MJ. Dental surgery in ant coagulated patients. Arch Intern Med 1998 ; 158 : 1610-6.
15 - Wahl MJ. Myths of dental surgery in patients receiving anticoagulant therapy. J Am Dent Assoc 2000; 131: 77-81.

16 - Campbell JH, Awaradot F, Murray RA. Anticoagulation and minor oral surgery: should the anticoagulation regimen is altered? J Oral Maxillofac Surg $2000 ; 58: 131-5$. médecine

buccale chirurgie buccale

VOL. $14, \mathrm{~N}^{\circ} 2$ 2008

page 109 